\title{
On-Demand Decode and Forward Cooperative MAC for VoIP in Wireless Mesh Networks
}

\author{
Kent Cheung, Li Li and Lajos Hanzo \\ School of ECS, University of Southampton, SO17 1BJ, UK. \\ Email: \{ktkc106, 115e08, lh\}@ecs.soton.ac.uk, http://www-mobile.ecs.soton.ac.uk
}

\begin{abstract}
The employment of wireless mesh networking in reallife scenarios has attracted substantial research interest in recent years and in this context VoIP has become a ubiquitous application. However, it has been demonstrated that VoIP transmissions over a multihop network may still remain inadequate in terms of their high packet-loss ratio and network-induced delays. To alleviate these limitations, we propose a novel distributed MAC-layer cooperation protocol, which is based on the decode-and-forward regime, whilst relying on the lowest possible control packet overhead. Furthermore, we employ several improvements across the protocol stack, including an improved PHY-layer, based on three-stage turbo-style differential detection, packet aggregation in the MAC-layer, as well as on adjusting the retransmission limit of each packet in order to reduce the delay imposed when employing cooperation. We characterize our improved system in a Wireless Mesh Network (WMN) scenario using the OMNeT++ network simulator and compare it to an 802.11g-based benchmarker. As a benefit of these techniques, we have observed up to 10 -fold reduction in the energy consumption per bit, despite increasing the number of simultaneous calls supported by up to 9 , when the number of hops between the sources and destination is 6 .
\end{abstract}

\section{INTRODUCTION}

Wireless mesh networking has attracted substantial interest in the research community in recent years [1] [2] [3]. A Wireless Mesh Network (WMN) consists of mesh clients and mesh routers, which dynamically configure themselves, while forming a mesh architecture. Additionally, the mesh routers may assume gateway/bridging functionality for the sake of connecting with other networks, such as cellular or sensor networks and the Internet [4]. They combine some of the benefits of ad-hoc and infrastructure-based networks, and thus they constitute an attractive model for flexible future wireless networks, especially since the architecture is congruent with the green communications philosophy of low-power multi-hop transmissions, which extends the conventional cellular coverage and facilitates flexible network expansion.

An ubiquitous application used in wireless networks is Voice over IP (VoIP). However, VoIP services tend to impose strict Quality of Service (QoS) requirements, such as maintaining a low Packet Loss Ratio (PLR) and delay, which is problematic in WMNs. Hence they require further research [5] [6]. In general, the performance of VoIP transmissions over WMNs is limited by factors, such as their inability to perform well both for excessively high or low node densities or for a high number of hops. The latter may be attributed to packet loss events due to interference, owing to transmissions over multiple hops, due to their high protocol overheads compared to the small VoIP payload size, etc. Maintaining a high VoIP transmission quality in the face of the potentially high end-to-end delay and packet loss probabilities imposed by multi-hop transmissions and user mobility, renders the provision of adequate voice quality in WMNs a substantial challenge. Similarly, providing reliable network routing that supports user mobility also remains a wide open research issue, since most solutions provide a slow response to a rapidly varying network topology. Additionally, supporting roaming and handovers with minimal disruption to the transmission quality remains a particular challenge [7] [8].

${ }^{1}$ This research has been funded by the Industrial Companies who are Members of the Mobile VCE, with additional financial support from the UK Government's Engineering \& Physical Sciences Research Council.
On a similar note, cooperative communications has attracted significant research attention over the past decade, as a benefit of its proficiency in mitigating the effects of the hostile wireless environment by exploiting the cooperative diversity provided by spatially separated antenna elements, which are capable of forming an uncorrelated Virtual-Multiple-Input-Multiple-Output (V-MIMO) system [9]. Some of their benefits are: a reduced outage probability, an increased rate region, reduced Bit Error Ratio (BER), etc. There are two main types of user cooperation, namely the Amplify-andForward (AF) and the Decode-and-Forward (DF) schemes [10]. In $\mathrm{AF}$, the relaying node receives and amplifies the source's transmissions based on an agreed power sharing between them, before forwarding it to the destination. This $\mathrm{AF}$ regime is less complex than DF, which requires that the relay node first decodes the source's transmissions, before re-encoding it and forwarding it to the destination. However, AF relaying has the effect of forwarding the amplified noise that was received at the relay.

In order to employ these cooperation aided schemes in a realistic network, higher layer cooperation-ready protocols must be designed for MAC and network-layer cooperation. For example, Liu et al. [11] designed a cooperative MAC protocol that establishes a list of potential relays and allows the source to send control packets to a selected relay in order to request its cooperation in forwarding its data packets. This scheme was later extended by Crismani et al. [12] to allow successive relaying, which reduces the multiplexing loss caused by the half-duplex constraint of practical wireless transceivers. Gharavi et al. [13] proposed a joint MAC layer and network layer cooperation aided protocol that selects a route to the destination, which facilitates the asynchronous distributed forwarding of the data packet by two nodes on a hop-by-hop basis using Distributed Linear Dispersion Codes (DLDC) [14].

Although the collection of cooperation-ready networking protocols is vast, only a few of them consider the particular applications that they are used for and the specific requirements that they impose. Motivated by the above discussions, in this contribution we study the viability of enabling cooperation in support of VoIP over a WMN using the IEEE $802.11 \mathrm{~g}$ standard and its improved versions. We demonstrate that owing to the previously mentioned factors the VoIP transmission quality attained in a WMN may remain inadequate. Explicitly, we propose a distributed decode-and-forward cooperative MAC protocol relying on the novel technique of initiative determination [15]. We specifically quantify the scalability of our proposed system by determining the maximum number of calls that it may support with satisfactory quality, as defined in [16] by measuring the average attainable $R$ Rating [17]. We show that our system provides improved scalability at a lower cost of transmit energy per information bit compared to a baseline system employing $802.11 \mathrm{~g}$ protocols. In contrast to similar 'score'-based relay selection schemes, such as those of [18] [19], our system is entirely distributed and does not rely on the exchange of control messages, thereby reducing the overhead imposed. Furthermore, in contrast to [20], we introduce cooperative forwarding, and consider a more realistic WMN scenario, consisting of randomly, rather than arbitrarily, distributed mobile nodes. We will demonstrate that the proposed cooperative regime is 
attractive for employment in VoIP communications over WMN and other strict-QoS multimedia services, confirming that cooperation constitutes an important paradigm in the context of future wireless networking.

The rest of the paper is organized as follows. In Section II, we detail our cooperative MAC protocol. Section III discusses the extra performance enhancements we proposed right across the protocol stack for assisting VoIP transmissions. In Section IV, we describe our simulation methodology and present our performance results. Finally, Section V provides our concluding remarks.

\section{CoOperative MAC-LAYER USING Initiative DETERMINATION}

We design a cooperation-aided MAC protocol for improving the VoIP transmission quality whilst minimizing the energy per bit for transmission over a multihop wireless network. Our goal is to reduce the transmission overhead, which is in contrast to the protocols described in the literature [11] [13] [18], which rely on exchanging control messages with the relaying nodes. We will demonstrate that our protocol effectively re-routes the packets without having to notify the network layer, should a momentary link failure occur due to excessive interference or deep channel fading, for example. We proceed by describing our cooperative protocol.

Firstly, we note that nodes complying with the 802.11 standard constantly monitor the channel in order to decode the MAC header of the packets, so that they can determine whether the packet was intended for them or not [21]. Thus, all nodes become aware of their environment without any need for extra control messaging. Exploiting this, each node can construct a table of MAC addresses from the decoded packet headers along with a time-stamp of when it was decoded and the power that the packet was received at as retrieved from the physical layer. Another consequence is that nodes can, provided that they are well-positioned, detect the occurrence of all link failures in their vicinity by inferring the lack of an ACKnowledgment packet from the link's destination. Thus, nodes that are aware that they are well-positioned and detect a link failure may opt for forwarding the unacknowledged packet, and they are likely to have a high probability of success, provided that the information used as the basis of its forwarding decision was reasonably reliable and recent.

In a network having a high user-density, it is likely that many nodes could act as potential relays, hence it becomes necessary to rank the relay candidates to avoid that they all simultaneously attempt to forward a specific packet, which would cause excessive interference at the destination, potentially rendering the forwarded copy unreliable. To accommodate multiple relay candidates, we introduce the notion of initiative determination, which we adopted from Vuran et al. [15], who used it as a binary indication of a node's willingness to route a packet based on fulfilling several criteria across multiple networking layers. We use the idea of initiative determination as a means of distributively deciding on the forwarding priorities of the candidate relay nodes by quantifying the likelihood of a successful forwarding operation on an integer scale. We term this the node's initiative rating, which we calculate as the ratio of the time $T_{h d}$, elapsed since the last packet heard from the destination, to the power $P_{r}$, received from the destination, given by $R_{i n i}=\left\lceil c \cdot T_{h d} / P_{r}\right\rceil$, where $c$ is a normalization factor.

Thus, a low $R_{i n i}$ indicates the presence of a high quality link between the current node and the destination that has a high chance of facilitating successful transmission, because the time elapsed since the last packet from the destination was heard is low and the received power of that packet was high. A higher $R_{i n i}$ would indicate either that the time duration since the last packet was heard is high (either the relaying or destination node may have moved to a distant location) and/or the received power was low. In either case, forwarding from this specific node would be unreliable. By setting a threshold value, $R_{t h}$, we can ensure that these unreliable nodes do not attempt to relay the packet. We will allocate the remaining nodes to the relay candidate (RC) set $\mathcal{R}$.

Let us now consider a link failure indicated by an ACKTimeOut, which occurs, when the system's delay exceeds the threshold, $A C K_{T O}$, which is formulated by $A C K_{T O}=\mathrm{SIFS}+$ $\tau_{\mathrm{ACK}}+\tau_{p}$, where SIFS represents the Short InterFrame Spacing parameter defined in the 802.11 standards, $\tau_{\mathrm{ACK}}$ is the transmission time of an ACK packet and $\tau_{p}$ is the estimated maximum propagation delay. After the RCs in $\mathcal{R}$ notice that there is a link failure, the RCs will enter a contention period and set their backoff timer to a value uniformly chosen in the interval $\left[1, R_{i n i}\right]$.

\begin{tabular}{|c|c|c|c|}
\hline \multirow[b]{2}{*}{ Source } & & & $\begin{array}{l}\text { ating-based } \\
\text { Backoff }\end{array}$ \\
\hline & 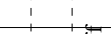 & Data & Data \\
\hline Destination & 10 & Datá & Data \\
\hline Relay Candidate 1 & $\begin{array}{lll}1 & 0 \\
1 & 0 & 0 \\
\end{array}$ &  & Data \\
\hline Relay Candidate 2 & 司: & \begin{tabular}{|l:l:l} 
Data & $\bar{n}$ & \multicolumn{1}{c}{} \\
\end{tabular} & $\begin{array}{l:}\text { Data } \\
\end{array}$ \\
\hline Relay Candidate 3 & $\bar{E}$ & $\begin{array}{ll}\text { Data } \\
\end{array}$ & \\
\hline Relay Candidate 4 & 10 & Datá1 & \\
\hline Data & - Transmitt & ing Data & \\
\hline Data & - Successfu & 1lly Receive Data & \\
\hline Datán-1 & - Failed to & Receive Data & \\
\hline
\end{tabular}

Fig. 1. Timing sequence of example transmission scenario.

As seen in the example of Fig. 1, the source's transmission is not received correctly by the intended destination, but it is correctly received by RC 1,2 and 3, which form the RC set $\mathcal{R}$. After noticing the lack of an acknowledgment from the destination, each node within $\mathcal{R}$ calculates its initiative rating for the specific destination considered based on its own table and then enters the contention phase. Let us assume that the RCs individually calculate the initiative ratings of $R_{i n i}=1,3,15$ at RC 1, 2 and 3 , respectively. If, in this example, $R_{t h}$ was set to 11 , then RC 3 would refrain from forwarding the packet, and only RC 1 and 2 will enter the contention phase. Let us assume furthermore that during the contention phase, RC 1 chooses a lower backoff value of say 1 , than RC 2 , so that it forwards the unacknowledged packet first. Upon noticing this, RC 2 refrains from forwarding the packet for the sake of avoiding any additional interference. In our modified protocol, the source's $A C K_{T O}{ }^{2}$ was extended to include the possibility of relaying, hence in this example the source notices that its data packet has been forwarded by RC 1 , hence it does not attempt to retransmit over its unreliable link.

It is important to note that the destination will not acknowledge the forwarded packet, since the intention of the forwarding node was to only assist the original source's transmission, thus the transmission of an ACK to the RC is meaningless. The data packet forwarded by the relay serves as an acknowledgment to the source to indicate that there was a RC nearby that forwarded the packet, while the transmission of an ACK to the source by the destination is not needed, since it is unlikely to be correctly received over the associated weak link anyway.

By using the initiative determination technique [15], we ensure that relaying actions will take place only when they are likely to succeed. Furthermore, the on-demand feature of the protocol refers to the fact that relaying is only activated if the destination could not correctly decode the packet, thus eliminating the potential wastage of energy by unnecessarily forwarding a packet. Naturally, RCs that failed to correctly decode the packet do not participate in the forwarding process at all.

${ }^{2}$ The new $A C K_{T O}$ at the source is given by $A C K_{T O}=2 \tau_{\text {DATA }}+$ SIFS $+\tau_{\mathrm{ACK}}+3 \tau_{p}+R_{t h} \sigma$, where $\tau_{\mathrm{DATA}}$ is the transmission time of the data packet, $R_{t h}$ is the previously mentioned rating threshold, and $\sigma$ is the slot duration of the MAC protocol. 


\section{Further Enhancements aCross the Protocol Stack}

Firstly, we augment the physical layer of our 802.11 system by using a near-capacity turbo detection/decoding scheme based on combining Soft-Input Soft-Output Multiple Symbol Differential Sphere Detection (SISO-MSDSD) with a Unity Rate Convolutional (URC) code and a Recursive Systematic Convolutional (RSC) code to form a three-stage concatenated system. This system has a BER turbo-cliff at an SNR value of $6.02 \mathrm{~dB}$ which is $0.82 \mathrm{~dB}$ from the capacity of $5.2 \mathrm{~dB}$. Thus our system exhitbits a nearcapacity performance. Since we invoke the receiver in the context of the OFDM scheme used in 802.11, we employ time-domain differentially-encoded OFDM, where the symbols allocated to each individual subcarrier are differentially encoded across the time-domain, i.e. between the same subcarrier of two consecutive OFDM symbols. Due to space limitation, we refer the interested reader to [22] for more information on turbo-style SISO-MSDSD detection, whilst details on the URC and RSC decoders can be found in [14] [23].

Additionally, we augment our MAC layer further by using packet aggregation [24] to reduce the excessive transmission overhead associated with short packets. In order to reduce the overhead imposed by the MAC layer (36 Bytes), we invoked the packet aggregation technique of [24] for the MAC Service Data Units (AMSDU), which requires that the receiver address of the constituent packets in the aggregate packet is the same. Each packet is extended by a 14 Byte header during the aggregation process, before attaching the standard 36 Byte MAC overhead, including the Cyclic Redundancy Check (CRC) sequence [24]. For example, if aggregating as few as two frames, the total MAC overhead is reduced from $2 \cdot 36=72$ Bytes to about 64 Bytes. Naturally, an increased aggregation gain may be attained, as the number of aggregated packets is increased. Furthermore, we reduce the retransmission limit in our system to ensure that the packet delay imposed does not become excessive, either due to the increased ACK-Timeout period used, or due to transmitting large packets as a consequence of the packet aggregation technique. It is more desirable to minimize the delay imposed at the expense of an increased packet loss ratio, since the packet losses may be mitigated by error concealment methods [25].

\section{Simulation Results and Discussions}

In this section, we describe our simulation methodology and present our results along with our related discussions.

\section{A. Simulation Methodology}

We compare the proposed system to a baseline $802.11 \mathrm{~g}$ basedWMN modeled in the OMNeT++ Network Simulator Tool [26] using the INETMANET package. The WMN is constructed by placing a fixed density of mesh clients in a $[d \times 200] \mathrm{m}$ area, where $d$ is $400 N_{\text {hops }}$ and $N_{\text {hops }}$ is the number of hops between the active nodes and the destination mesh router, which is placed at the point $(d \mathrm{~m}, 100 \mathrm{~m})$. Furthermore, a variable number of active VoIP mesh clients are placed at $(0 \mathrm{~m}, y)$, where $y$ is a uniformly distributed random variable in the range $[0,200] \mathrm{m}$. We assume that the active mesh clients establish VoIP calls to users within a separate network and that the destination mesh router at the opposite end of the simulated network is capable of carrying out the gateway/bridging functionality. Thus, the VoIP data packets must flow between the active mesh clients and the destination mesh router using one or more hops, depending on their separation distance. This forms a typical wireless mesh network topology, which employs multihop routing and tends to exhibit a bottleneck at the backhaul nodes, as shown in Fig. 2. Additionally, the inactive mesh clients undergo node mobility, which is modeled using a constant walking speed of $2 \mathrm{~m} / \mathrm{s}$ and a random direction (angle) that is uniformly distributed.
Should any node attempt to move outside the simulation area, it will be reflected back from the outer perimeter at an identical speed and an angle of reflection equal to the angle of incidence.

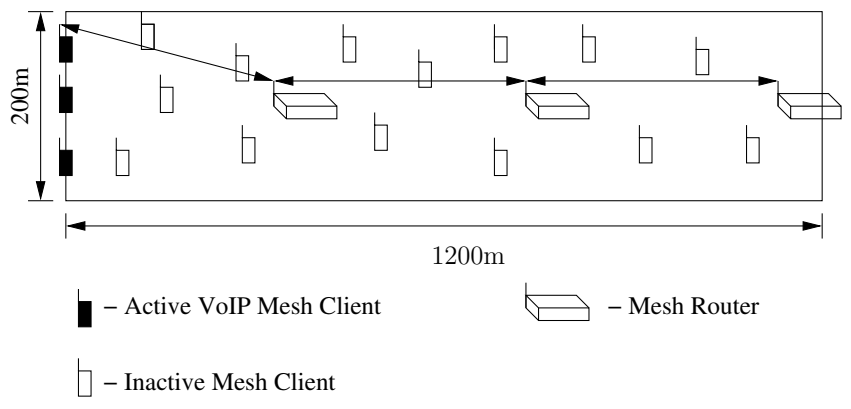

Fig. 2. The active mesh clients communicate with the destination mesh router by using the backhaul mesh routers to route their packets in the simulated wireless mesh network scenario. This example illustrates the case when $N_{\text {hops }}=3$.

To specifically target the scalability issue in wireless mesh networks, we evaluate the performance of our systems by increasing the number of simultaneous calls placed over a duration of 10 minutes and at increasing $N_{\text {hops }}$ from the destination mesh router. Accordingly, we employ the Adaptive Multi Rate WideBand (AMR-WB) speech codec [25] in the application layer of our traffic-generating nodes, where, for simplicity, we emulate the codec's functions by generating Constant Bit Rate (CBR) traffic from each of the active mesh clients to the mesh router, and vice versa. Therefore, assuming that we use the $23.85 \mathrm{kbps}$ mode of the codec and generating 480 -bit packets every $20 \mathrm{~ms}$, the packet length is about 60 bytes. Each speech packet is accompanied by a 12-byte RTP header and an 8-byte UDP header, before being passed down to the network layer.

In the network layer, we use predetermined routes to ensure that the VoIP packets are routed accordingly to the $N_{\text {hops }}$ under investigation. As a further improvement of the system, we use Robust Header Compression (RoHC) [27]. Normally, the protocol overhead consisting of RTP/UDP/IP headers totals 40 bytes and this substantially reduces the efficiency of the system. RoHC sets up a state machine at both the transmitter and the receiver, which are kept synchronized by transmitting only the slowly varying header fields, as suggested in [27]. This effectively reduces the overhead needed per VoIP packet, but we have to mention that if the compressed header becomes corrupted, a full header has to be transmitted. For the sake of fair comparisons, we employ RoHC in all test scenarios, since this may be used, regardless whether cooperation is activated or not. Furthermore, for simplicity we also assume that RoHC is capable of reducing the RTP/UDP/IP headers to a constant length throughout the simulations and that the state machines at both ends are perfectly synchronized.

In the physical layer of the baseline $802.11 \mathrm{~g}$ benchmarker simulations, we fixed the modulation and coding modes to Quadrature Phase Shift Keying (QPSK) and 1/2 rate Recursive Systematic Convolutional (RSC) coding, which is a mode of operation that appears in the standards, so that it was comparable to our enhanced system using SISO-MSDSD, which uses Differential QPSK in conjunction with the same $1 / 2$ rate RSC code. Since the symbol duration of the $802.11 \mathrm{~g}$ transceivers is $4 \mu \mathrm{s}$ at a symbol-rate of $250 \mathrm{ksymbols} / \mathrm{sec}$ and there are $48 \mathrm{OFDM}$ subcarriers per OFDM symbol, the bitrate using 4-level modulation combined with $1 / 2$ rate RSC coding becomes $24 \mathrm{Mbps}$. In the coherent detection aided standard system, zero-forcing channel equalization was performed assuming perfect channel knowledge [21].

Additionally, in order to make the simulations tractable, we abstract the physical layer of our system using a lookup table method. Firstly, we evaluate the performance of our physical layer 
TABLE I

MAIN Simulation PARAMETERS

\begin{tabular}{|l|l|}
\hline Simulation Parameter & Value \\
\hline Channel Bandwidth & $20 \mathrm{MHz}$ \\
Path Loss Model & Free Space Path Loss \\
Path Loss Exponent & 2 \\
Fast Fading Model & Correlated Rayleigh Fading \\
Doppler Frequency & 0.01 \\
Inactive Mesh Client Speed & $2 \mathrm{mps}$ \\
Symbol Rate & $250 \mathrm{ksymbols} / \mathrm{s}$ \\
No. of OFDM subcarriers & 48 \\
Transmit Power & $2 \mathrm{~mW}$ \\
Thermal Noise & $-110 \mathrm{dBm}$ \\
Receiver Sensitivity & $-90 \mathrm{dBm}$ \\
Modulation Order & 4 \\
Outer Decoder & $1 / 2 \mathrm{Rate}$ RSC with $M=6$ \\
No. of Outer Decoding Iterations & 9 \\
Inner Decoder & URC \\
No. of Inner Decoding Iterations & 2 \\
SISO-MSDSD Window Size & 6 \\
Cooperative MAC Constant $c$ & $10-4$ \\
Cooperative MAC $R_{t h}$ & 31 \\
Cooperative MAC Retry Limit & 3 \\
RoHC Header Size & $14 \mathrm{~B}$ \\
Speech Codec & AMR-WB \\
Speech Codec Mode & $23.85 \mathrm{kbps}$ \\
Speech Packet Length & $60 \mathrm{~B}$ \\
Speech Packet Period & $20 \mathrm{~ms}$ \\
Inactive Mesh Client Density & $200 \mathrm{nodes} / \mathrm{km}^{2}$ \\
\hline
\end{tabular}

schemes by estimating their BER performance over a correlated fading channel and over a large range of interference plus noise power, whilst assuming that the imposed interference is Gaussian. These BER values are then used to generate the estimated PERs for a given received SINR value and frame length, $N_{f}$, using $\operatorname{PER}(S I N R)=1-(1-B E R(S I N R))^{N_{f}}$. These lookup tables are then used in the OMNeT++ simulations instead of modeling the behavior of the transceivers bit-by-bit, which we found to substantially reduce the simulation times, potentially by a few orders of magnitude. However, during the simulations, we incorporated the effects of path loss, which was calculated by the free-space path loss equation, given by $\lambda^{2} / 16 \pi^{2} d^{\alpha}$, where $\lambda$ is the wavelength of the carrier, $d$ is the distance between the communicating entities, and $\alpha$ is the free-space path-loss exponent. We summarize the main simulation parameters in Table I.

\section{B. Simulation Results and Discussions}

Using the simulation methodology presented in Section IV-A, we compare our cooperative system to the non-cooperative standard $802.11 \mathrm{~g}$ system. The results presented here were recorded by averaging over 20 different random seeds and for different numbers of simultaneous VoIP calls, $n$, and $N_{\text {hops }}$, where each run lasts for a simulated time of $600 \mathrm{sec}$.

Noting that an R-Rating of over 80 is considered satisfactory [16], we compare the call capacity of a standard 802.11gbased WMN and our cooperation aided WMN. In Fig. 3, we observe that the number of simultaneous calls supported by the baseline system decreases rapidly upon increasing $N_{\text {hops }}$, whereas the cooperation aided system maintains a gracefully decreasing number of simultaneous calls, as $N_{\text {hops }}$ increases. These effects may be attributed to the lower PLR and delays suffered in the cooperation aided system, which may be explained as follows.

Firstly, the near-capacity three-stage turbo-style detector and decoder employing the SISO-MSDSD algorithm, as well as the URC and RSC decoders facilitates strong error correction, provided that the received SINR is higher than $6.02 \mathrm{~dB}$. This means that as the number of hops and the number of simultaneous calls increases and hence the level of intraflow interference becomes high, the physical layer may still provide for adequate link capabilities.

In the case that the link is severely affected by the level of interference in the system, the cooperative protocol ensures that a relay candidate forwards the packet almost immediately without any need for control messages. The destination is likely to receive this forwarded packet, since the relay candidate that forwarded it has a low initiative rating, which indicates a high link reliability. Since the number of retransmissions in the cooperative system is limited to three, when the original link is weak and no reliable relay candidates can be found, the packets destined for that particular transmission are dropped sooner than in the non-cooperative case. This is desirable, since it clears the transmit buffer for that source node, thus preventing that node from imposing additional interference onto other ongoing transmissions.

Another beneficial effect is that because the packet is effectively re-routed using decode-and-forward, the receive SINR for that packet is higher than that for the original transmission from the source. This value of SINR may be much higher than the original owing to the reduced distance between the relay and destination, and the effect of the inverse square pathloss law. The new 'virtual' path that is created with the aid of the relaying node can be used as long as necessary or until the channel conditions between the original source and destination improve, because the amount of interference imposed on the destination by other ongoing transmissions is reduced. Not only does this manifest itself in a reduced PLR and delay, but additionally provides some secondary benefits, such as a reduced probability of buffer overflow in the MAC layer as an advantage of the A-MSDU as well as the prompt re-routing or dropping of packets that are unlikely to be successfully transmitted.

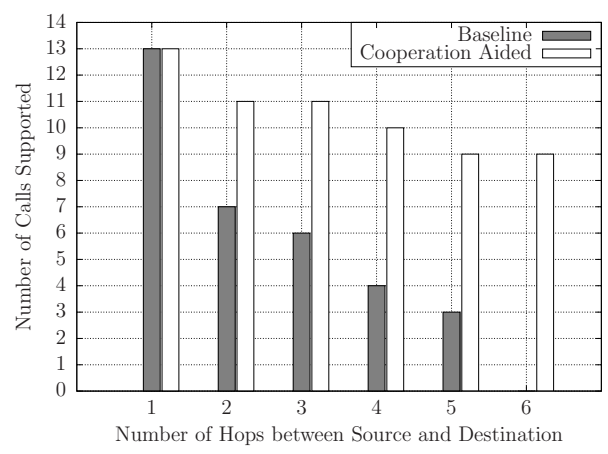

Fig. 3. Average number of simultaneous calls supported when using the standard $802.11 \mathrm{~g}$ and our cooperation-aided 802.11 schemes versus $N_{\text {hops }}$ between the source and the destination using the parameters of Table I.

Fig. 4 shows the energy-cost of communicating a single application layer data bit in terms of RF transmit energy, when simulating $n=3,6,9,12$ or 15 simultaneous calls using the standard $802.11 \mathrm{~g}$-based WMN and our cooperation aided WMN, which we calculate using the parameters of Table I to arrive at the Energy Consumption Ratio (ECR) defined as ECR $=$ (Total Transmit Time $\times$ Transmit Power)/(Total No. of Application Data Bits Received). Observe from Fig. 4 that the cooperative scheme outperforms the non-cooperative benchmark scheme in terms of service provision capabilities, despite reducing the energy per bit to an almost stable figure for up $n=9$ simultaneous calls for each value of $N_{\text {hops }}$, whilst for the baseline system, the ECR increases sharply for both an increasing number of simultaneous calls and the value of $N_{\text {hops }}$. The beneficial effects of re-routing invoked for avoiding retransmission over an unreliable link combined with the A-MSDU technique allowed us to reduce the overall transmission overhead. Furthermore, our improved physical layer employing turbo-style MSDSD-URC-RSC also enhanced the overall energy efficiency of the system. estimation of the non-cooperative coherent system was perfect. In a realistic scenario, this is not the case, which would result in an even higher performance benefit for our proposed solution. 


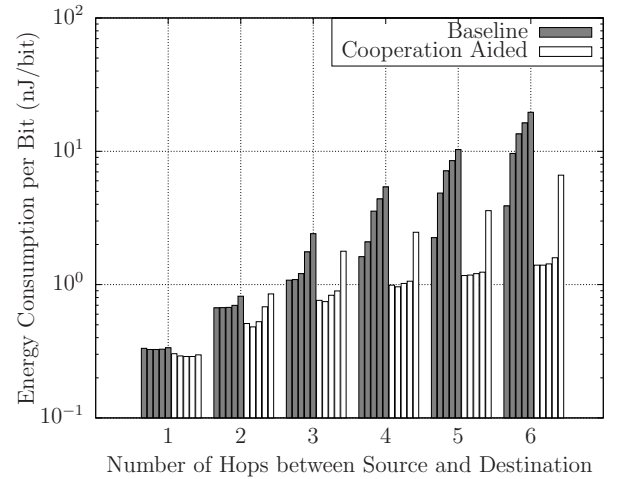

Fig. 4. Average energy consumption per information bit for $n=3,6$, $9,12,15$ simultaneous calls when using the standard $802.11 \mathrm{~g}$ and our cooperation-aided 802.11 schemes versus $N_{\text {hops }}$ between the source and the destination using the parameters of Table I.

\section{CONCLUSIONS}

For the sake of improving the VoIP transmission quality of an 802.11-based WMN, we introduced several improvements across the protocol stack, whilst concurrently reducing the energy cost per bit. The more notable amendments include the employment of a cooperation-aided MAC protocol, an improved physical layer based on differentially encoded modulation and turbo-style MSDSD, as well as MSDU packet aggregation. As a benefit of these techniques, we have observed an up to 10 -fold reduction in the energy consumption per bit, whilst attaining a significantly higher service provision capability of up to $n=9$ number of simultaneous calls when using $N_{\text {hops }}=6$ hops between the VoIP call's source and destination. From these results, we can conclude that our cooperation aided system specifically improves the scalability of WMNs, so that they may become viable in real-world scenarios.

\section{ACKNOWLEDGEMENTS}

The work reported in this paper has formed part of the Green Radio Research Programme of the Virtual Centre of Excellence in Mobile \& Personal Communications, Mobile VCE, www.mobilevce.com. Fully detailed technical reports on this research are available to Industrial Members of Mobile VCE.

\section{REFERENCES}

[1] L. Ding, T. Melodia, S. Batalama, and J. Matyjas, "Distributed routing, relay selection, and spectrum allocation in cognitive and cooperative ad hoc networks," IEEE Communications Society Conference on Sensor Mesh and Ad Hoc Communications and Networks (SECON'10), Boston, MA USA, pp. 1-9, Jun. 2010.

[2] X. Shao, C. Hua, and A. Huang, "Robust resource allocation for end-to-end rate guarantee in wireless mesh networks," in IEEE International Conference on Communications (ICC'10), Cape Town, South Africa, May 2010, pp. 1-6.

[3] Y. Song, C. Zhang, and Y. Fang, "Stochastic traffic engineering in multihop cognitive wireless mesh networks," IEEE Transactions on Mobile Computing, vol. 9, no. 3, pp. 305-316, Mar. 2010.

[4] I. Akyildiz and X. Wang, "A survey on wireless mesh networks," IEEE Communications Magazine, vol. 43, no. 9, pp. 23-30, Sept. 2005.

[5] S. Ganguly, V. Navda, K. Kim, A. Kashyap, D. Niculescu, R. Izmailov, S. Hong, and S. Das, "Performance optimizations for deploying VoIP services in mesh networks," IEEE Journal on Selected Areas in Communications, vol. 24, no. 11, pp. 2147-2158, Nov. 2006.

[6] S. Garg and M. Kappes, "An experimental study of throughput for UDP and VoIP traffic in IEEE 802.11b networks," in IEEE Wireless Communications and Networking (WCNC'03), New Orleans, LA, vol. 3, Mar. 2003, pp. 1748-1753.

[7] X. Wang, A. Patil, and W. Wang, "VoIP over wireless mesh networks: Challenges and approaches," in ACM International Conference on Wireless Internet (WICON'06), Boston, MA USA, vol. 220, no. 6, Aug. 2006.

[8] D. Niculescu, S. Ganguly, K. Kim, and R. Izmailov, "Performance of VoIP in a 802.11 wireless mesh network," in IEEE International Conference on Computer Communications (INFOCOM'06), Barcelona, Spain, Apr. 2006, pp. 1-11.
[9] A. Sendonaris, E. Erkip, and B. Aazhang, "User cooperation diversity. Part I-II," IEEE Transactions on Communications, vol. 51, no. 11, pp. 1927-1948, Nov. 2003.

[10] J. Laneman, D. Tse, and G. Wornell, "Cooperative diversity in wireless networks: Efficient protocols and outage behavior," IEEE Transactions on Information Theory, vol. 50, no. 12, pp. 3062-3080, Dec. 2004

[11] P. Liu, Z. Tao, S. Narayanan, T. Korakis, and S. S. Panwar, "CoopMAC: A cooperative MAC for wireless LANs," IEEE Journal on Selected Areas in Communications, vol. 25, no. 2, pp. 340-354, Feb. 2007.

[12] A. Crismani, F. Babich, and L. Hanzo, "Cross-layer solutions for cooperative medium access control protocols," in IEEE Vehicular Technology Conference (VTC'10S), May. 2010, pp. 1-5.

[13] H. Gharavi, B. Hu, and N. Wu, "A design framework for highdensity wireless ad-hoc networks achieving cooperative diversity," IEEE International Conference on Communications (ICC'10), Cape Town, South Africa, pp. 1-5, May 2010.

[14] L. Hanzo, O. Alamri, M. El-Hajjar, and N. Wu, Near-capacity Multifunctional MIMO Systems: Sphere-packing, Iterative Detection and Cooperation, 1st ed. Wiley-IEEE Press, 2009.

[15] M. Vuran and I. Akyildiz, "XLP: A cross-layer protocol for efficient communication in wireless sensor networks," IEEE Transactions on Mobile Computing, vol. 9, no. 11, pp. 1578-1591, Nov. 2010.

[16] ITU-T - Series G: Transmission systems and media, digital systems and networks - International telephone connections and cicuits Transmission planning and the E-model - G.107: The E-model: a computational model for use in transmission planning. [Online]. Available: http://www.itu.int/rec/T-REC-G.107-200904-I

[17] ITU-T - Series G: Transmission systems and media, digital systems and networks - Quality of service and performance - Generic and user-related aspects - G.1070: Opinion model for videotelephony applications. [Online]. Available: http://www.itu.int/rec/TREC-G.1070-200704-I

[18] A. Bletsas, A. Lippnian, and D. Reed, "A simple distributed method for relay selection in cooperative diversity wireless networks, based on reciprocity and channel measurements," in IEEE Vehicular Technology Conference (VTC'05S), Stockholm, Sweden, vol. 3, May 2005, pp. 1484-1488.

[19] Z. Bali, W. Ajib, and H. Boujemaa, "Distributed relay selection strategy based on source-relay channel," in IEEE International Conference on Telecommunications (ICT'10), Doha, Qatar, Apr. 2010, pp. 138142.

[20] K. Kim and S. Hong, "VoMESH: voice over wireless MESH networks," in IEEE Wireless Communications and Networking Conference (WCNC'06), Las Vegas, NV USA, vol. 1, Apr. 2006, pp. 193-198.

[21] IEEE Standard for Information technology Telecommunications and information exchange between systems Local and metropolitan area networks Specific requirements Part 11: Wireless LAN Medium Access Control (MAC) and Physical Layer (PHY) Specifications Amendment 4: Further Higher Data Rate Extension in the $2.4 \mathrm{GHz}$ Band. [Online]. Available: http://standards.ieee.org/getieee802/802.11.html

[22] V. Pauli, L. Lampe, and R. Schober, "Turbo DPSK using soft multiple-symbol differential sphere decoding," IEEE Transactions on Information Theory, vol. 52, no. 4, pp. 1385-1398, Apr. 2006.

[23] L. Hanzo, Y. Akhtman, L. Wang, and M. Jiang, MIMO-OFDM for LTE, WiFi and WiMAX: Coherent versus Non-coherent and Cooperative Turbo Transceivers, 1st ed. Wiley-IEEE Press, 2010.

[24] D. Skordoulis, Q. Ni, H.-H. Chen, A. Stephens, C. Liu, and A. Jamalipour, "IEEE 802.11n MAC frame aggregation mechanisms for next-generation high-throughput WLANs," IEEE Wireless Communications, vol. 15, no. 1, pp. 40-47, Feb. 2008.

[25] L. Hanzo, F. Somerville, and J. Woodard, Voice and Audio Compression for Wireless Communications, 2nd ed. Wiley-IEEE Press, 2007.

[26] A. Varga and R. Hornig, "An overview of the OMNeT++ simulation environment," in Proc. 1st international conference on Simulation tools and techniques for communications, networks and systems \& workshops Simutools '08, 2008, pp. 1-10.

[27] P. Fortuna and M. Ricardo, "Header compressed VoIP in IEEE 802.11," IEEE Wireless Communications, vol. 16, no. 3, pp. 69-75, Jun. 2009. 